\title{
DIFERENTES DOSAGENS DE PÓ INERTE E TEMPERATURAS EM MILHO ARMAZENADO PARA CONTROLE DE GORGULHO-DO-MILHO
}

\section{DIFFERENT DOSAGES OF INERT DUST AND TEMPERATURES IN STORAGE CORN FOR CONTROL OF MAIZE WEEVIL}

\author{
Maria Aparecida Braga CANEPPELE ${ }^{1}$ \\ Patrícia de Jesus ANDRADE ${ }^{2}$ \\ Alexandre Garcia SANTAELLA ${ }^{3}$
}

\begin{abstract}
RESUMO
No Brasil, Sitophilus zeamais Mots. e S. oryzae L. (Coleoptera: Curculionidae) estão entre os insetos - praga de maior importância para os grãos de milho e arroz armazenados, respectivamente, provocando perdas quantitativas e qualitativas. A terra de diatomácea tem sido estudada por diversos pesquisadores visando à proteção de grãos armazenados. Esta pesquisa objetivou avaliar a eficácia do pó inerte em diferentes dosagens e temperaturas para o controle de $S$. zeamais em grãos de milho armazenados. Amostras de $400 \mathrm{~g}$ de milho foram acondicionadas em frascos de vidro com tampas teladas e submetidas aos seguintes tratamentos: pó inerte nas dosagens de $0,125,500$ e $750 \mathrm{~g} \mathrm{t}^{-1}$ nas temperaturas de 25,30 e $35^{\circ}$ $\mathrm{C}$, em cinco repetiç̃es. Cada frasco recebeu 30 insetos adultos de $S$. zeamais para as avaliações da mortalidade com três,

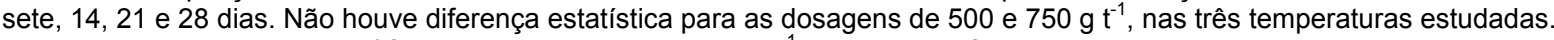
Nas temperaturas de 25 e $30{ }^{\circ} \mathrm{C}$ as dosagens de 500 e $750 \mathrm{~g} \mathrm{t}^{-1}$ se tornaram eficientes a partir do sétimo dia de avaliação. Na temperatura de $35{ }^{\circ} \mathrm{C}$ as três dosagens, no terceiro dia de avaliação, provocaram quase $100 \%$ de mortalidade. A terra diatomácea é eficaz para o controle de $S$. zeamais, sendo a dose de $500 \mathrm{~g} \mathrm{t}^{-1}$ indicada para o controle dessa praga em temperaturas acima de $25^{\circ} \mathrm{C}$.

Palavras-Chave: grãos armazenados; mortalidade; estratégias de controle.
\end{abstract}

ABSTRACT
In Brazil, Sitophilus zeamais Mots. and S. oryzae L. (Coleoptera: Curculionidae) are among the most important insects for the corn grains and stored rice, respectively, causing quantitative and qualitative losses. The diatomaceous earth has been studied by several researchers, for the protection of stored grains. The research aimed to evaluate the effectiveness of the inert dust in different dosages and temperatures for the $S$. zeamais' control in grains of stored corn. Samples of $400 \mathrm{~g}$ of corn were packed in glass flasks with lid covered by nylon fabric and submitted to the following treatments: Inert dust in the dosages of $0,125,500$ and $750 \mathrm{~g} \mathrm{t}^{-1}$ upon temperatures of 25,30 and $35^{\circ} \mathrm{C}$, in five replicates. Each flask received 30 adult insects of $S$. zeamais for the mortality evaluations with three, seven, 14,21 and 28 days. There were not statistical difference for the dosages of 500 and $750{\mathrm{~g} . \mathrm{t}^{-1}}^{-1}$ in the three studied temperatures. On temperatures of 25 and $30^{\circ} \mathrm{C}$ the dosages of 500 and $750 \mathrm{~g} \mathrm{t}^{-1}$ became efficient from the seventh evaluation day. In the temperature of $35^{\circ} \mathrm{C}$ the three dosages, on third evaluation day, obtained almost $100 \%$ of mortality. The diatomaceous earth demonstrated as effective treatment in the control of $S$. zeamais. The $500 \mathrm{~g} \mathrm{t}^{-1}$ dosage can be recommended for the control of $S$. zeamais on stored corn in temperatures above $25^{\circ} \mathrm{C}$.

Key-words: stored grains; mortality; control strategies.

\footnotetext{
${ }^{1} \mathrm{Dr}^{\mathrm{a}}$. em Ciências Biológicas, Núcleo de Tecnologia em Armazenagem, Faculdade de Agronomia e Medicina Veterinária, Universidade Federal de Mato Grosso, Av. Fernando Correa da Costa, 2367, CEP: 78060-900, Cuiabá, MT. E-mail: canepele@terra.com.br

${ }^{2}$ Estudante de Mestrado do Programa de Pós-Graduação em Agricultura Tropical, Universidade Federal de Mato Grosso, Cuiabá-MT, Brasil. Email: patriciajandrade@gmail.com.

${ }^{3}$ Estudante de Agronomia, Universidade Federal de Mato Grosso, Cuiabá-MT, Brasil. E-mail: santaella.agro@gmail.com.
} 
CANEPPELE, M. A. B. et al. Diferentes dosagens de pó inerte e ...

\section{INTRODUÇÃO}

A qualidade dos grãos e sementes de milho, em especial a qualidade fisiológica, pode ser afetada pela ação de diferentes agentes, entre eles insetos-praga, como o Sitophilus zeamais Mots. (Coleoptera: Curculionidae), que corresponde a um dos grandes responsáveis pela deterioração de grãos e sementes armazenadas (Rees, 1996).

O S. zeamais trata-se de uma praga primária, por causar danos aos grãos sadios e intactos enquanto as larvas se alimentam em seu interior. A fase de pupa ocorre também no interior do grão e o adulto, ao emergir, saindo do grão deixando orifícios (Evans, 1981). Infesta principalmente milho, trigo, arroz e sorgo tendo preferência para ovipositar em milho e trigo, podendo, também, se desenvolver em produtos cereais processados como macarrão ou mandioca desidratada (Dobie et al.,1984). A temperatura ideal para o seu desenvolvimento é $28{ }^{\circ} \mathrm{C}$, embora seja possível se desenvolver entre 15 e $34^{\circ} \mathrm{C}$.

No Brasil, $S$. zeamais e $S$. oryzae estão entre os insetos de maior importância para os grãos de milho e arroz armazenados, respectivamente, provocando perdas quantitativas e qualitativas, que podem atingir até $15 \%$ do peso e ainda comprometerem a qualidade nutritiva do milho (Santos et al. 1983). Ambas as espécies são encontradas em todas as regiões quentes e tropicais do mundo e podem infestar os grãos no campo antes do armazenamento (Fontes et al., 2003). A perda de massa de grãos foi comprovada por Marsaro-Junior (2005) pela infestação por $S$. zeamais em diferentes variedades de milho, na ordem de $3,33 \%$ a $6,25 \%$. As alterações da qualidade devem-se a presença de fungos e insetos que contaminam a massa de grãos. Assim a infestação por $S$. zeamais é um risco potencializador de danos econômicos.

O controle de pragas em produtos armazenados é feito, preferencialmente, com fumigantes liquefeitos ou solidificados a exemplo da fosfina. Entretanto, a utilização indevida da fosfina levou ao surgimento de populações de insetos resistentes e à detecção de resíduos em grãos e sementes. A partir de tal fato, novos métodos de aplicação e de distribuição do fumigante tem sido testados, como a adição de dióxido de carbono e nitrogênio (Faroni, 1997). A utilização de terra de diatomácea, como inseticida alternativo, é uma forma de controlar sem desencadear problemas provocados pelos inseticidas químicos.

A terra diatomácea é um depósito geológico que consiste de esqueletos petrificados de numerosas espécies de silícios e organismos unicelulares marinhos e outras algas. É um produto natural, estável, não produz resíduos químicos tóxicos e não reage com outras substâncias (Korunic, 1998) e tem sido utilizada visando a proteção de grãos armazenados (Nielsen, 1998). Os insetos em contato com a terra diatomácea morrem devido à desidratação pela perda de água da camada de lipídios da epicutícula, associado a temperaturas acima de $20^{\circ} \mathrm{C}$ (Korunic, 1998; Ceruti et al., 2008).

O tratamento de grãos com terra de diatomáceas possui algumas vantagens em relação ao tratamento químico convencional, tais como: a) controle das diversas pragas que atacam sementes armazenadas; b) longo efeito residual na semente; c) substituição de inseticidas químicos, tanto os preventivos como os curativos (expurgo), durante o armazenamento da semente; d) controle de populações de pragas resistentes aos inseticidas químicos e não promoção de resistência em insetos. Trata-se de produto seguro para o usuário e de efeito inseticida duradouro (Lorini et al., 2003).

O objetivo da pesquisa foi testar a eficácia de diferentes dosagens de terra de diatomácea nas temperaturas de 25,30 e $35^{\circ} \mathrm{C}$, em condições de laboratório, para o controle de $S$. zeamais em grãos de milho.

\section{MATERIAL E MÉTODOS}

O experimento foi conduzido no Núcleo de Tecnologia em Armazenagem da Universidade Federal de Mato Grosso. Foi conduzido em câmaras B.O.D (Biological Organism Development), reguladas nas temperatura de 25,30 e $35{ }^{\circ} \mathrm{C}$, umidade relativa do ar de $65 \pm 5 \%$ e $12 \mathrm{~h}$ de fotoperíodo. A terra diatomácea utilizada para os experimentos foi o produto comercial Insecto ${ }^{\circledR}$, pó seco, solto, insolúvel em água e isento de matérias estranhas.

O delineamento experimental foi inteiramente casualizado, em esquema fatorial $4 \times 3$ x 5 (doses $x$ temperaturas x período de avaliação), com cinco repetições. Foram utilizadas as seguintes doses: 1) Testemunha, sem adição de Insecto ${ }^{\circledR}$; 2) Dose $0,05 \mathrm{~g}$ de Insecto ${ }^{\circledR}$ para cada $400 \mathrm{~g}$ de milho o que corresponde a $125 \mathrm{~g} \mathrm{t}^{-1}$; 3) Dose de $0,2 \mathrm{~g}$ por $400 \mathrm{~g}$ de milho o que corresponde a $500 \mathrm{~g} \mathrm{t}^{-1} ; 4$ ) Dose de $0,3 \mathrm{~g}$ por $400 \mathrm{~g}$ de milho o que corresponde a $750 \mathrm{~g} \mathrm{t}^{-1}$.

As diferentes dosagens do produto foram homogeneizadas com grãos de milho limpo seco e com $14 \%$ de conteúdo de água. Cinco repetições de $400 \mathrm{~g}$ de grãos foram usados para cada tratamento, colocadas em frascos de vidro com tampa telada. Em cada frasco foram colocados 30 insetos adultos, não sexados de $S$. zeamais de sete a 14 dias de idade. Os tratamentos foram mantidos nas câmaras e observados durante 28 dias para registro de mortalidade.

Para todos os tratamentos, a mortalidade foi avaliada no terceiro, sétimo, $14^{\circ}, 21^{\circ}$ e $28^{\circ}$ dia. Os insetos foram considerados mortos quando não se moveram ao serem tocados por um pincel e expostos a luz de uma luminária por dois minutos.

Foi efetuada a análise de variância, e as médias foram comparadas pelo teste de Tukey a $5 \%$ de probabilidade.

\section{RESULTADOS E DISCUSSÃO}

Em todas as temperaturas e dosagens testadas foi observado o efeito inseticida da terra 
CANEPPELE, M. A. B. et al. Diferentes dosagens de pó inerte e ...

diatomácea (Tabela 1). A mortalidade iniciou já no terceiro dia após o tratamento com a terra diatomácea nas três temperaturas estudadas. As doses de 500 e $750 \mathrm{~g} \mathrm{t}^{-1}$ não apresentaram diferenças significativas entre si, para as três temperaturas, independente do período de avaliação. Para a maior temperatura não houve diferença na mortalidade de $S$. zeamais entre as três doses do estudadas e também a partir dos 14 dias na testemunha.

TABELA 1 - Valores médios de mortalidade (\%) de S.zeamais em diferentes doses de terra de diatomácea e temperaturas, em 28 dias.

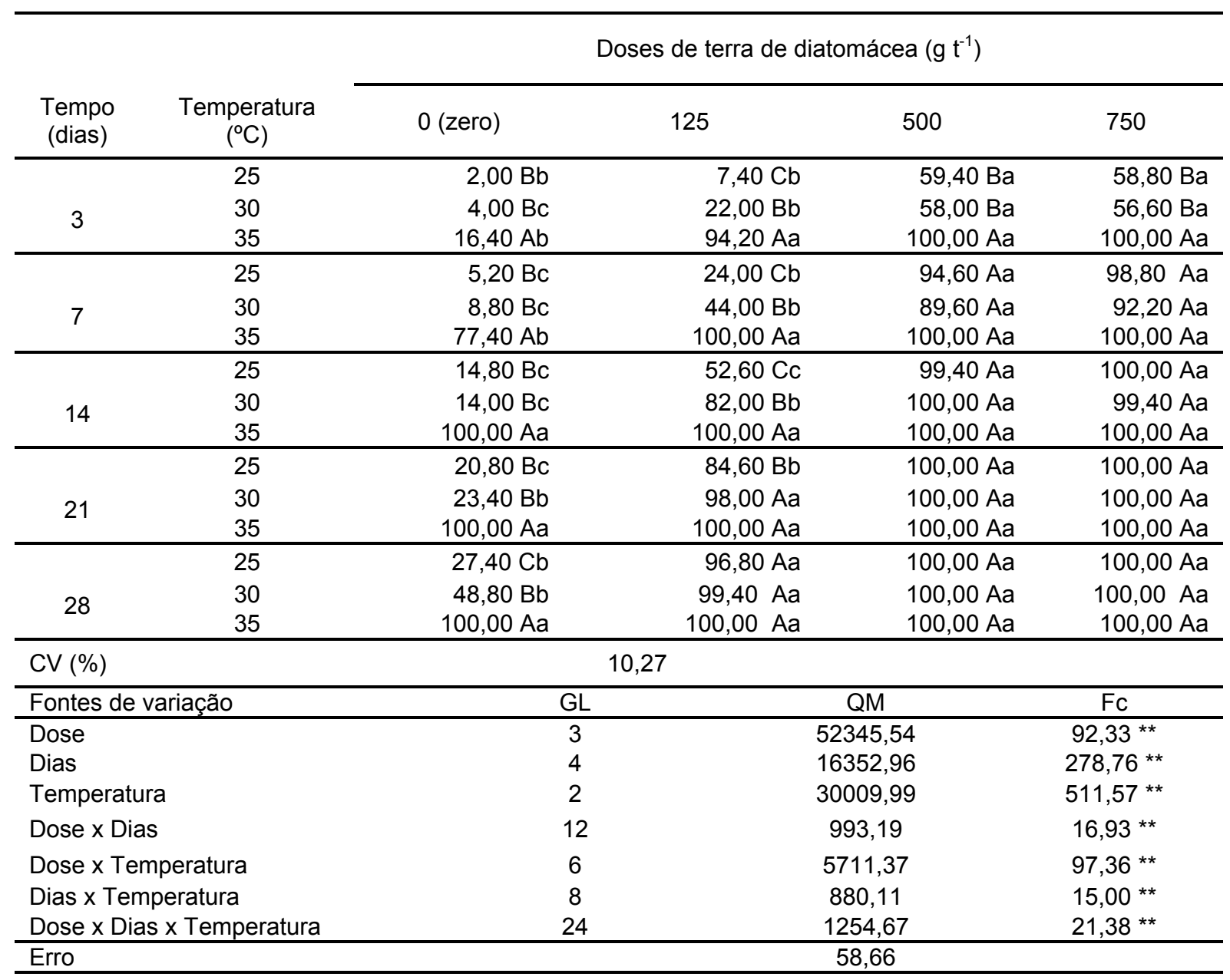

Médias seguidas da mesma letra maiúscula nas colunas e minúscula nas linhas, em cada período de avaliação, não diferem entre si, pelo teste de Tukey a $5 \%$ de probabilidade. $F^{*}=$ significativo a $5 \%$. $F^{* *}=$ significativo a $1 \%$.

Na temperatura de $25^{\circ} \mathrm{C}$, no terceiro dia a mortalidade dos insetos atingiu $60 \%$ para as dosagens de 500 e $750 \mathrm{~g} \mathrm{t}^{-1}$, com sete dias a mortalidade ficou próximo de $100 \%$. Na testemunha a mortalidade foi inferior a $30 \%$ durante os 28 dias de duração do experimento (Tabela 1). Para a dosagem de $125 \mathrm{~g} \mathrm{t}^{-1}$, é necessário um tempo de exposição acima de 21 dias para atingir a mortalidade máxima.

Ceruti et al. (2008) observaram que na temperatura de $25{ }^{\circ} \mathrm{C}$ para as dosagens de 500 e $750 \mathrm{~g} \mathrm{t}^{-1}$ não diferiram quanto a mortalidade de $S$. zeamais e que a testemunha não atingiu $73,3 \pm 6,5 \%$ de mortalidade durante os 28 dias de avaliação.

Os resultados obtidos estão em acordo com Pinto Jr. (2008) e Lorini et al. (2002), que acompanharam o desenvolvimento de diferentes pragas de grãos armazenados em milho tratado com pó inerte e observaram um efeito interativo entre dosagem e período de exposição do inseto ao pó inerte. Esses autores observaram que em períodos maiores de exposição, as dosagens mais elevadas proporcionaram melhor controle da população.

Para a temperatura de $30{ }^{\circ} \mathrm{C}$, as dosagens de 500 e $750 \mathrm{~g} \mathrm{t}^{-1}$ atingiram $100 \%$ de mortalidade só aos 14 dias (Tabela 1). O mesmo foi observado por Demissie et al. (2008), avaliando o controle de $S$. zeamais em três genótipos de milho. Já na dosagem de $125 \mathrm{~g} \mathrm{t}^{-1}$ ocorreu mortalidade acima de $80 \%$. Na testemunha a mortalidade estava foi inferior a $15 \%$, nesse mesmo período de 
CANEPPELE, M. A. B. et al. Diferentes dosagens de pó inerte e ...

observação. O mesmo foi verificado por MarsaroJunior et al. (2006) avaliando a eficiência de terra diatomácea no controle de $S$. zeamais em milho armazenado em Roraima, quando observaram redução da eficiência do pó inerte com a redução da dosagem em curto período de observação.

$\mathrm{Na}$ temperatura de $35^{\circ} \mathrm{C}$ ao terceiro dia, a dosagem de $125 \mathrm{~g} \mathrm{t}^{-1}$, não diferiu de 500 e $750 \mathrm{~g} \mathrm{t}^{-1}$ apresentando mortalidade próxima de 100\%. A testemunha após o $14^{\circ}$ dia não diferiu dos demais tratamentos. Nessa temperatura houve maior eficiência da mortalidade do inseto. Para as maiores dosagens a taxa de $100 \%$ de mortalidade foi atingida aos 14 dias (Tabela 1). Temperaturas próximas de $27{ }^{\circ} \mathrm{C}$ são adequadas para o bom desenvolvimento do inseto, podendo explicar a alta mortalidade em temperatura superior como a $35^{\circ} \mathrm{C}$. As temperaturas ótimas para o desenvolvimento da maioria dos insetos de produtos armazenados estão na faixa de 25 à $33{ }^{\circ} \mathrm{C}$ (Fields, 1992). Na testemunha também ocorreu uma alta porcentagem de mortalidade, isto pode ter acontecido, porque em temperatura superior a $30^{\circ} \mathrm{C}$ a perda de água nos insetos é mais intensa, ocorrendo desidratação e morte.

Athanassiou et al. (2005) observaram em trigo armazenado, tratados com diferentes dosagens de pó inerte aplicadas em $S$. oryzae e
Tribolium confusum, que a mortalidade foi de $100 \%$ para S. oryzae após um período de $48 \mathrm{~h}$ a $32{ }^{\circ} \mathrm{C}$ em qualquer das doses aplicadas. Esses resultados evidenciam a atuação do pó inerte somada a temperaturas superiores a $32{ }^{\circ} \mathrm{C}$ no favorece o controle deses insetos, estando de acordo com os dados obtidos para a faixa de temperatura de $35^{\circ} \mathrm{C}$ neste experimento (Tabela 1).

A mortalidade do $S$. zeamais foi significativamente afetada pelo tempo de exposição (Tabela 2). Para a testemunha essa foi maior quanto maior o tempo de exposição, não ocorrendo diferenças a partir do $21^{\circ} \mathrm{dia}$, independente da temperatura. Com o uso da terra essa tendência se manteve para a dosagem de $125 \mathrm{~g} \mathrm{t}^{-1}$ e para $500 \mathrm{e}$ $750 \mathrm{~g} \mathrm{t}^{-1}$ não houve diferença na mortalidade a partir do sétimo dia de exposição. $\mathrm{Na}$ temperatura de $35^{\circ} \mathrm{C}$ a partir do terceiro dia a mortalidade dos insetos não diferiu e atingiu o máximo, independente da dosagem, já para a testemunha isso ocorreu no $14^{\circ}$ dia de exposição. As condições de armazenamento de um produto, como temperatura e umidade relativa, influenciam na mortalidade dos insetos adultos e nas progênies, como observado por Sthaters et al. (2002) utilizando a terra de diatomácea no controle de diferentes espécies de inseto-praga.

TABELA 2 - Valores médios de mortalidade (\%) de S. zeamais durante 28 dias, em diferentes doses de terra de diatomácea e temperaturas.

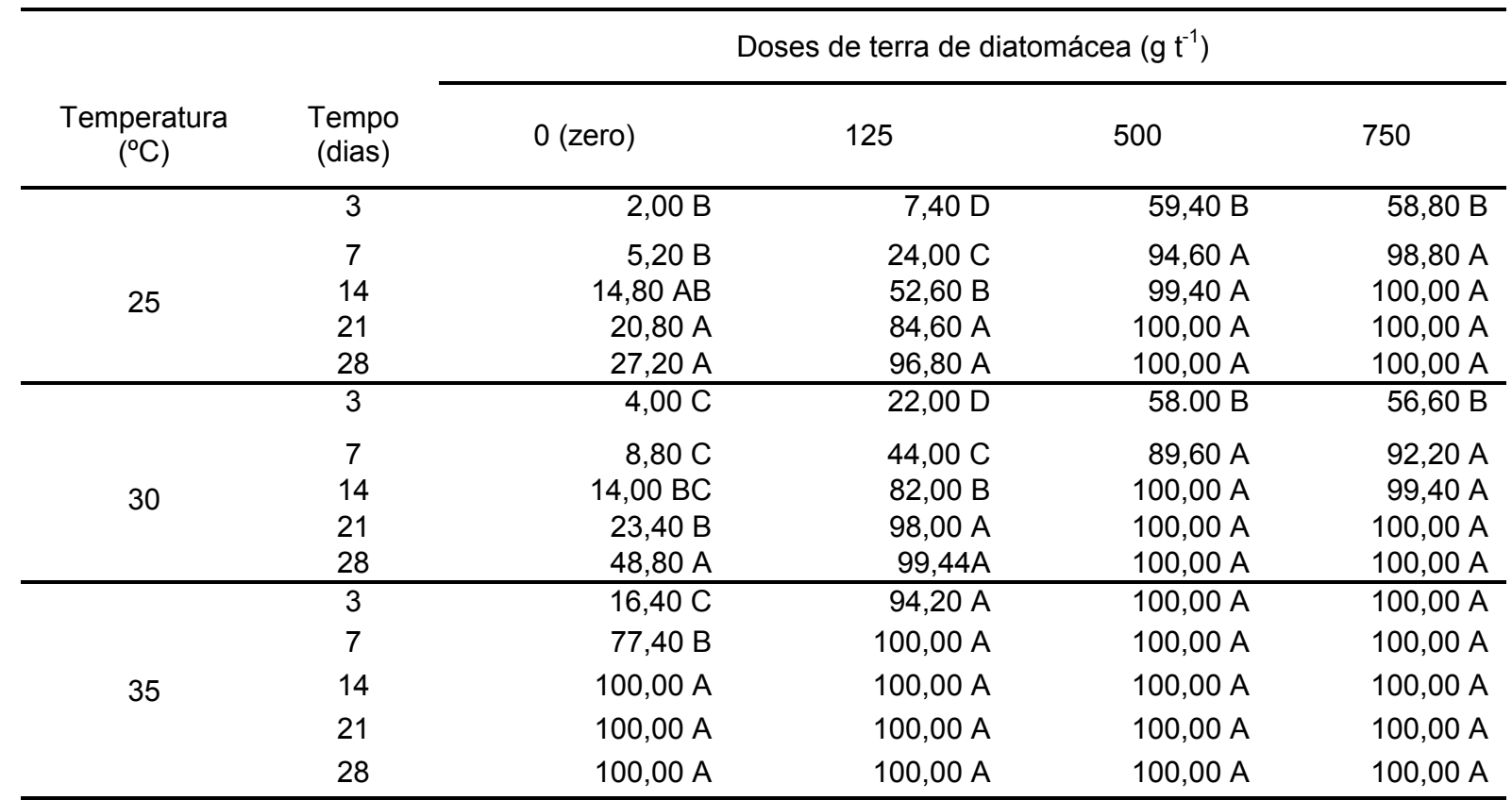

Médias seguidas da mesma letra maiúscula nas colunas, para cada dose e em cada temperatura, não diferem entre si, pelo teste de Tukey a $5 \%$ de probabilidade. $\mathrm{F}^{*}=$ significativo a $5 \% . \mathrm{F}^{* *}=$ significativo a $1 \%$.

As terras diatomáceas, Insecto $^{\circledR}$ e Keepdry ${ }^{\circledR}$, proporcionaram 99,7\% e 99\%, respectivamente, de mortalidade em estudo realizado com milho armazenado tratado com terra diatomácea (Smiderle \& Cícero, 1999). No entanto, não foram detectados descendentes da população dos insetos avaliados. Esse fato sugere que os produtos, mesmo não tendo eficiência de $100 \%$ de mortalidade, impediram que os insetos provocassem danos ou mesmo postura nas 
CANEPPELE, M. A. B. et al. Diferentes dosagens de pó inerte e ...

sementes. Desta forma, as perspectivas de uso da terra diatomácea no tratamento das sementes de milho são promissoras, tendo em vista que não foram constatados prejuízos provocados pelos produtos aplicados nas sementes.

\section{CONCLUSÕES}

Os resultados desta pesquisa permitem inferir que: i) A interação entre doses de terra de diatomácea e temperatura proporciona melhor eficiência de controle em S. zeamais; ii) A dosagem de $500 \mathrm{~g} \mathrm{t}^{-1}$ pode ser recomendada para o controle de $S$. zeamais em milho armazenado em temperaturas superiores a $25^{\circ} \mathrm{C}$; iii) A $35^{\circ} \mathrm{C}$ as três dosagens foram eficientes no terceiro dia de avaliação; iv) Dosagens de terra de diatomácea, acima de $500 \mathrm{~g} \mathrm{t}^{-1}$ apresentam melhor eficiência na mortalidade dos insetos, independente da temperatura.

\section{CONFLITOS DE INTERESSE}

O produto comercial testado neste trabalho foi doado pela Empresa Bernardo Química S.A. ao Núcleo de Tecnologia de Armazenagem da UFMT para a realização da pesquisa. Os autores declaram que não possuem subordinação de consultoria, financiamento ou vinculação empregatícia à empresa produtora do mesmo.

\section{REFERÊNCIAS}

1. ATHANASSIOU, C. G. et al. Insecticidal efficacy of diatomaceous earth against Sitophilus oryzae (L.) (Coleoptera: Curculionidae) and Tribolium confusum du Val (Coleoptera) on stored wheat: influence of dose rate, temperature and exposure interval. Journal of Stored Products Research, v. 41, n. 1, p. 47-55, 2005.

2. CERUTI, F. C. et al. Eficácia de terra diatomácea e temperatura para o controle do gorgulho-do-milho em milho armazenado em milho. Scientia Agraria, v. 9, n. 1, p. 73-78, 2008.

3. DEMISSIE, G.; TEFERAB, T.; TADESSE, A.; Efficacy of Silicosec, filter cake and wood ash against the maize weevil, Sitophilus zeamais Motschulsky (Coleoptera: Curculionidae) on three maize genotypes. Journal of Stored Products Research, v. 44, n. 3, p. 227-31, 2008.

4. DOBIE, P. et al. Insects and arachnids of tropical stored products, their biology and identification: a training manual. London: Tropical Development and Research Institute, 1984.

5. EVANS, D. E. The biology of stored products Coleoptera. In: CHAMP, B. R., HIGHLEY, E. (Eds.). Proceedings of the Australian Development Assistance Course on the Preservation of Stored Cereals. Canberra: CSIRO Division of Entomology, 1981, p. 149-185.

6. FARONI, L. R. A. Principais pragas de grãos armazenados. In: ALMEIDA, F. A. C.; HARA, T.; CAVALCANTI MATA, M. E. R. M. Armazenamento de grãos e sementes nas propriedades rurais. Campina Grande: UFPB/SBEA, 1997. p.189291.

7. FIELDS, P. G., The control of stored-product insects and mites with extreme temperatures. Journal of Stored Products Research, v. 28, n. 2, p. 89-118, 1992.

8. FONTES L. S.; ALMEIDA FILHO, A. J; ARTHUR, V. Danos causados por Sitophilus oryzae (Linné, 1763) e Sitophilus zeamais Motschulsky, 1855 (Coleoptera: Curculionidae) em cultivares de arroz (Oryza sativa L.) Arquivos do Instituto Biológico, v. 70, n. 3, p. 303-307, 2003.

9. KORUNIC, Z. Rapid assessment of the insecticidal value of diatomaceous earths without conducting bioassays. Journal of Stored Products Research, v. 33, n. 3, p. 219-229, 1997

10. KORUNIC, Z. Review diatomaceous earths, a group of natural insecticides. Journal of Stored Products Research, v. 34, n. $2 / 3$, p. $87-97,1998$.

11. KORUNIC, Z. et al. Grain bulk density as affected by diatomaceous earth and application method. Postharvest Biology and Technology, v. 13, n. 1, p. 81-89, 1998.

12. LORINI, I., et al. Tratamento de sementes armazenadas com pós inertes à base de terra de diatomáceas. Passo Fundo: Embrapa Trigo, 2003. 4 p. (Embrapa Trigo. Comunicado Técnico online, 113). Disponível em: <http:// www.cnpt.embrapa.br/biblio/co/p_co113.htm>. Acesso em 22 ago. 2005.

13. MARSARO-JUNIOR, A. L. et al; Effectiveness of different dosages of diatomaceous earth to control Sitophilus zeamais (Coleoptera: Curculionidae). In corn stored in the state of Roraima; In: INTERNATIONAL WORKING CONFERENCE ON STORED PRODUCT PROTECTION, 9., 2006, Campinas, Proceedings... Campinas: Associação Brasileira de PósColheita, 2006. p. 1264-1268.

14. MARSARO-JUNIOR, A. L. et al. Avaliação da resistência de híbridos de milho ao ataque de Sitophilus zeamais Motschulsky (Coleoptera: Curculionidae) no grão armazenado. Semina, v. 26, n. 2, p. 165-170, 2005

15. NIELSEN, P. S. The effect of a diatomaceous earth formulation on the larvae of Ephestia kuehniella Zeller. Journal of Stored Products Research, v. 34, n. 2/3, p. 113-121, 1998.

16. PINTO JUNIOR, A. R. Eficiência de terra de diatomáceas no controle de Sitophilus oryzae (L), Cryptolestes ferrugineus (Stephens), Tribolium castaneum (Herbst) em milho armazenado a granel. Revista da Faculdade de Zootecnia, Veterinária e Agronomia (Uruguaiana), v. 15, n. 1, p. 61-70, 2008.

17. REES, D. P. Coleoptera. In: SUBRAMANYAN, B.; HAGSTRUM, D. W. Integrated management of insects in stored products. New York: Marcel Dekker, 1996. p.1-39.

18. SMIDERLE, O. J; CíCERO, S. M. Tratamento inseticida e qualidade de sementes de milho durante o armazenamento. Scientia Agricola, v. 56, n. 4, p. 1245-1254, 1999.

19. STATHERS, T. E.; DENNIFF, M.; GOLOB, P. The efficacy and persistence of diatomaceous earth admixed with commodity against four tropical stored product beetle pests. Journal of Stored Products Research, v. 40, n. 1, p. 113123, 2004.

Recebido em 06/01/2009 Aceito em 30/07/2010 
